\title{
Analytical Formula of Nonlinear Interference in Few-Mode Fibers in Strong Coupling Regime
}

\author{
Abdallah A. I. Ali ${ }^{1}$, Abdulaziz E. El-Fiqi ${ }^{1}$, Ziad A. El-Sahn ${ }^{2}$, \\ Hossam M. H. Shalaby ${ }^{1,2}$, and Rameash K. Pokharel ${ }^{3}$ \\ ${ }^{1}$ Egypt-Japan University of Science and Technology (E-JUST), Alexandria 21934, Egypt \\ ${ }^{2}$ Photonics Group, Alexandria University, Alexandria 21544, Egypt \\ ${ }^{3}$ E-JUST Center, Kyushu University, Fukuoka 819-0395, Japan \\ abdallah.ali@ejust.edu.eg, abdulaziz.elfiqi@ejust.edu.eg, ziad.elsahn@ieee.org, shalaby@ieee.org, \\ pokharel@ed.kyushu-u.ac.jp
}

\begin{abstract}
We derive a closed-form formula for the nonlinear interference in few-mode fibers (FMFs) in strong coupling regime. We also formulate an expression for the nonlinear FMFs capacity. This is carried out by extending the Gaussian noise model (GN-model), that has been used with single-mode fibers (SMFs), to FMFs. We inspect the derived formulas over a mode-division multiplexing (MDM) system that carries wavelength-division multiplexed (WDM) signals on each polarization of every spatial mode. The nonlinear coupling among three co-propagated modes reduces the average optical signal-to-noise ratio (OSNR) by about $11.5 \mathrm{~dB}$ when compared to single-mode propagation. Also, a differential mode group delay (DMGD) of $300 \mathrm{ps} / \mathrm{km}$ between the fundamental mode $\mathrm{LP}_{01}$ and the other two modes $\mathrm{Lp}_{11 a(b)}$ reduces the nonlinearity penalty by about $10 \%$.
\end{abstract}

Keywords: Few-mode fibers (FMFs), four-wave mixing (FWM), Gaussian noise model, modedivision multiplexing (MDM), nonlinearity modeling.

\section{Introduction}

As single-mode fibers (SMFs) are approaching their nonlinear capacity limit in the near future, space-division multiplexing (SDM) is becoming the next-generation candidate that increases the capacity per fiber [1]. SDM systems use the space as a new dimension. This is normally carried out by multiplexing the data using either different modes of a FMF (called MDM) or different cores of a multicore fiber. However, fiber nonlinearity is an important limitation in long haul transmission systems [2]. Therefore, studying the nonlinear interaction in FMFs is crucial to evaluate the viability of MDM for long haul transmission. Good efforts have already been made to model the impacts of nonlinear propagation effects on the performance of SMF systems. One of these models is the GN-model [3], which name refers to the main assumption of the Gaussianality of both the signal and nonlinear interference. The GN-model assumes that the signal is divided into spectral components, and then uses a four-wave mixing (FWM)-like approach to consider the different nonlinearity impacts [3]. A limited number of studies have been conducted on the nonlinearity in FMFs [2,4-6]. In [5], a model has been proposed for evaluating the nonlinearity in FMFs. In [6], we have derived a simple analytical formula for addressing the nonlinear interference in FMFs in case of no coupling among spatial modes. In practice, the linear coupling or crosstalk among spatial modes cannot to be avoided [7].

In this paper, we extend our work in [6] by considering the strong coupling among spatial modes. Specifically, we derive a simple analytical formula, that measures the impact of nonlinearity on the system performance in the presence of coupling in FMFs, and develop a new expression for the nonlinear capacity of FMFs. Using our developed formula, we evaluate the impact of nonlinearity on both optical signal-to-noise ratio (OSNR) and maximum reach. The effect of differential mode group delay (DMGD) is considered in our evaluation as well. In addition, we demonstrate the FMFs capacity for different modulation formats and the trade-off between the capacity and the maximum reach of FMFs.

\section{GN-Model for FMFs in Strong Coupling Regime}

The frequency domain generalized Manakov equation that describes the electric field propagation through FMFs at the presence of strong coupling among spatial modes can be written as in [7] as 
follows:

$$
\frac{\partial \vec{E}}{\partial z}=\alpha \vec{E}+j \frac{\omega^{2}}{2} \overrightarrow{\beta_{2}} \vec{E}+j \gamma \kappa \vec{E} * \vec{E}^{\star} * \vec{E}, \quad \kappa=\sum_{p \leq q}^{N} \frac{32}{2^{\delta_{p q}}} \frac{f_{p q}}{6 N(2 N+1)}
$$

where $\vec{E}$ is a $2 N$ vector that represents the electric fields in the frequency domain for $N$ spatial modes with two polarizations, $z$ is the propagation distance, $\alpha$ is the attenuation constant and is assumed equal for all modes, $\omega$ is the angular frequency, $\vec{E}^{\star}$ refers to the conjugate of vector $\vec{E}$, and $*$ refers to the convolution process. The average group velocity dispersion (GVD) $\overline{\beta_{2}}$ is equal to the summation of the GVDs for all modes divided by $2 N, \gamma$ is identical to the nonlinear coefficient of SMFs, and $\delta_{p q}$ is the Kronecker delta function. $f_{p q} \stackrel{\text { def }}{=}\left(A_{e f f} / I_{p} I_{q}\right) \iint\left|F_{p}(x, y)\right|^{2}\left|F_{q}(x, y)\right|^{2} d x d y$ represents the inter-modal nonlinear coupling coefficient between spatial modes $p$ and $q$ in case of $p \neq q$ and intra-modal nonlinear coupling coefficient in case of $p=q$, where $F_{p}(x, y)$ is the spatial mode profile function for mode with index $p, \mathrm{~A}_{e f f}$ is the effective area of the fundamental mode, and $I_{p}=\iint\left|F_{p}(x, y)\right|^{2} d x d y$.

The right-hand side of (1) is divided into two parts, the first is the linear part including the attenuation and dispersion, and the second is the nonlinear part due to Kerr effect in FMFs. The last term in (1) is a double convolution and is assumed as an independent source term. The solution of that differential equation is divided into a linear part (i.e., the solution of the equation in case of no nonlinearity) and a nonlinear part. The nonlinear solution is difficult to be obtained directly. Therefore, we use the GN-model for obtaining a perturbative solution for that part.

The GN-model is a FWM-like model based on dividing the signal spectrum into spectral components and expressing the nonlinearity in a way similar to the classical formula of FWM [3]. Here, we adapt the GN-model, already used with SMFs, to FMFs and follow a similar procedure as in [3] to obtain an analytical expression for the nonlinear interference. The model assumes that the transmitted signal on each mode is a WDM signal modeled as a Gaussian noise-like-signal with independent spectral components. In addition, the effect of the nonlinear interference (NLI) on the transmitted signal is modeled as an additive white Gaussian noise (AWGN) which is statistically independent from the amplified spontaneous emission (ASE) noise. The OSNR can thus be written as $O S N R=P_{t x, p} /\left(P_{A S E}+P_{N L}\right)$, where $P_{t x, p}$ is the transmitted power per mode, $P_{A S E}$ is ASE noise power and $P_{N L}$ is the NLI power, which is estimated by integrating its power spectral density (PSD) $Q_{N L}(f)$ over the noise bandwidth as $P_{N L}=\int Q_{N L}(f) d f$.

After a rigorous mathematical analysis, $Q_{N L}(f)$ is obtained by statistically averaging the power of the nonlinear electrical field, then applying the signal and model assumptions. At Nyquist case, where the channel bandwidth $B_{c h}$ is equal to both the sampling rate $R_{s}$ and the channel spacing $\Delta f$, we formulate a closed-form expression for the NLI as a noise power per mode at the center channel of the WDM, that is given by:

$$
P_{N L, p}=\frac{3}{16} N_{s}\left(\gamma \kappa L_{\mathrm{eff}}\right)^{2}\left(\frac{P}{N B_{c h}}\right)^{3} \sum_{h}\left(\frac{\operatorname{arcsinh}\left(\psi^{+}\right)+\operatorname{arcsinh}\left(\psi^{-}\right)}{\pi \beta_{2 h} L_{e f f}}\right) B_{n},
$$

where

$$
\psi^{ \pm}=\frac{\sqrt{3}}{4} \pi L_{\mathrm{eff}} B_{\omega}\left(\frac{\sqrt{3}}{2} \pi \beta_{2 h} B_{\omega} \pm \Delta \beta_{1_{p h}}\right),
$$

$\Delta \beta_{1 p h}=\beta_{1 h}-\beta_{1 p}$ is the DMGD between modes with indices $(p, h)$, and $\beta_{1 h}$ and $\beta_{1 p}$ are the inverse of group velocity for mode with indices $p$ and $h$, respectively. $\beta_{2 h}$ is the GVD for mode with index $h$ in $\mathrm{ps}^{2} / \mathrm{km}, P$ is the total transmitted power, $L_{\mathrm{eff}}=\left(1-e^{-\alpha L_{s}}\right) / \alpha$ is the effective length, $B_{n}$ is the noise bandwidth, and $B_{\omega}=N_{c h} B_{c h}$ is the total WDM bandwidth. Also $N_{s}, N$, and $N_{c h}$, are the number of spans, spatial modes, and WDM channels per mode, respectively.

By assuming matched electrical filtering at the receiver and that the received signal is free of intersymbol interference (ISI), we can get the electrical signal-to-noise ratio (SNR) using the relation $S N R=\left(B_{n} / R_{S}\right) O S N R$. By applying the SNR expression to the capacity formula for polarizationmultiplexed (PM) optical channels $C=2 \frac{R_{s}}{\Delta f} \log _{2}(1+S N R)$ [8], we get a closed-form expression for the capacity of PM-FMFs as

$$
C=2 \sum_{p} \log _{2}\left(1+\frac{P_{t x, p}}{B_{n} N_{s} F(G-1) h v+P_{N L, p}}\right),
$$

where $F$ is the optical amplifier noise figure, $h$ is plank's constant, $v$ is the center channel frequency, and $G$ is the amplifier gain. 


\section{Model Results}

The proposed model is evaluated over a MDM system using FMFs supporting three spatial modes $\mathrm{LP}_{01}, \mathrm{LP}_{11 a}$, and $\mathrm{LP}_{11 b}$, each with dual polarization states as in [9]. The dispersion coefficient equals $19.3 \mathrm{ps} / \mathrm{nm} . \mathrm{km}$ for $\mathrm{LP}_{01}$ mode and equals $18.3 \mathrm{ps} / \mathrm{nm} . \mathrm{km}$ for both $\mathrm{LP}_{11 a}$ and $\mathrm{LP}_{11 b}$ at $1540 \mathrm{~nm}$ reference wavelength. The nonlinear coefficient $\gamma=1.77 \mathrm{~W}^{-1} \mathrm{~km}^{-1}$ and the nonlinear coefficient tensor $\mathrm{f}_{p q}[9]$ is listed in Table 1 . The attenuation coefficient is $\alpha=0.226 \mathrm{~dB} / \mathrm{km}$.

Table 1. Non-zero calculated values of $\mathrm{f}_{p q}$ for three modes: $\mathrm{LP}_{01}, \mathrm{LP}_{11 a}$, and $\mathrm{LP}_{11 b}$.

\begin{tabular}{|c|c|c|c|}
\hline & $\mathrm{LP}_{10}$ & $\mathrm{LP}_{11 a}$ & $\mathrm{LP}_{11 b}$ \\
\hline $\mathrm{LP}_{10}$ & 1 & 0.496 & 0.496 \\
$\mathrm{LP}_{11 a}$ & 0.496 & 0.747 & 0.249 \\
$\mathrm{LP}_{11 b}$ & 0.496 & 0.249 & 0.747 \\
\hline
\end{tabular}

The fiber is divided into ten spans, each with $100 \mathrm{~km}$ length. The span loss is completely compensated using ideal few-mode erbium-doped fiber amplifiers (EDFAs) with $6 \mathrm{~dB}$ noise figure located at the end of each span. Each spatial mode carries nine WDM channels at $R_{s}=32$ Gbaud and channel bandwidth $B_{c h}=32 \mathrm{GHz}$. Each WDM channel carries two QPSK signals through polarizationdivision multiplexing (PDM) with $100 \mathrm{~Gb} / \mathrm{s}$ data rate plus $28 \%$ for forward error correction (FEC) and network protocols overhead. We assume that the dispersion is compensated at the receiver using digital signal processing to isolate the effect of nonlinearity on the system performance.

Figure 1 shows the nonlinear penalty impacts on the OSNR and the maximum length that FMFs can reach for different number of co-propagating modes (1, 2, and 3). In Fig. 1-a, the effect of nonlinear interaction between modes becomes more significant by increasing the transmitted power. When, the transmitted power exceeds $5 \mathrm{dBm}$, the average OSNR (for three co-propagating modes) is decreased by about $7 \mathrm{~dB}$ when compared with single-mode propagation (only $\mathrm{LP}_{01}$ ). Furthermore, the increase of DMGM to $300 \mathrm{ps} / \mathrm{km}$ (between the fundamental mode $\mathrm{LP}_{01}$ and the other two modes $\mathrm{Lp}_{11 a(b)}$ ) reduces the nonlinear penalty by approximately $2 \mathrm{~dB}$ compared to the zero DMGD case. Fig.1-b shows a similar behavior in terms of the maximum reach at $\mathrm{BER}=10^{-3}$ before FEC. It is a clear that the DMGM increases the maximum reach that can be achieved by FMFs.
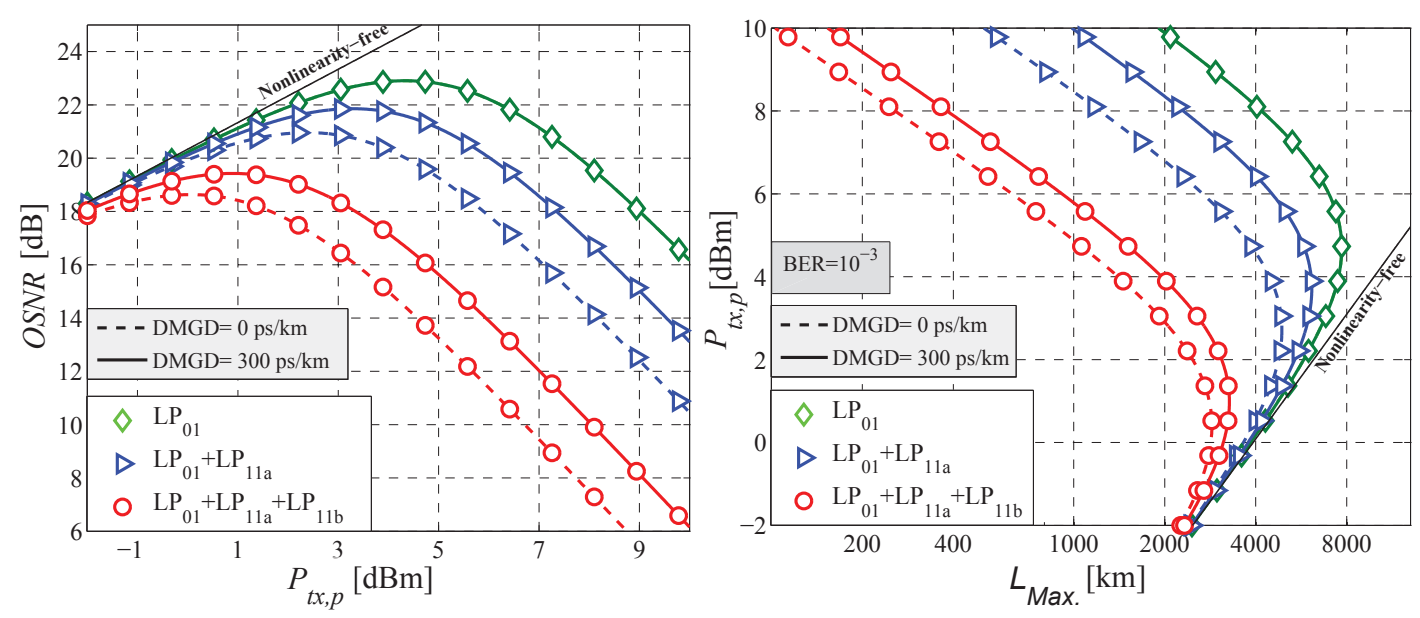

Fig. 1. a) OSNR versus launch power per mode over FMFs $\left(\mathrm{LP}_{01}, \mathrm{LP}_{11 a, b}\right)$, b) maximum reach versus launch power per mode for different number of propagating modes at two distinct DMGD cases.

Figure 2 shows the effect of transmitted power and reach on the system capacity for different constellation levels: QPSK, 16-QAM, and 64-QAM. From Fig. 2-a, it is clear that the impact of nonlinearity is negligible at low constellation levels but it becomes significant at high constellation levels. The top curve (diamond) shows the maximum theoretical capacity that the system can support under nonlinearity regardless of the constellation level. The results in Fig. 2-b shows the trade-off between distance and capacity for different modulation formats. Increasing the level of the constellation can achieve a higher capacity, but over a shorter distance. 

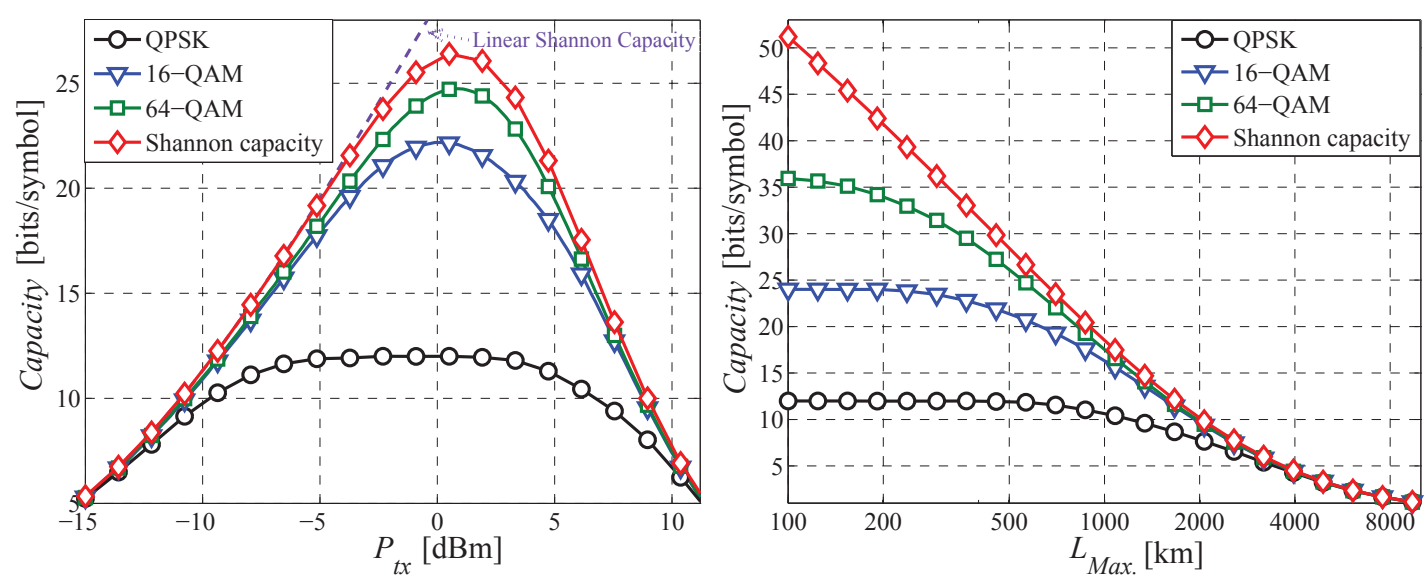

Fig. 2. a) Capacity versus transmitted power for different modulation techniques, b) capacity versus fiber length for different modulation techniques.

\section{Conclusion}

We have extended the GN-model for FMFs to address the impacts of nonlinearity penalty in strong coupling regime. A closed-form formula for NLI power has been derived and an expression for nonlinear capacity of strong-coupled FMFs has been introduced. Using the proposed model, we have verified that the DMGD reduces the impact of nonlinearity on the system performance. In addition, we have quantified the tradeoff between the capacity and the maximum reach when limited by the nonlinear coupling among spatial modes.

\section{Acknowledgement}

This work was supported by the Ministry of Higher Education (MoHE), Egypt.

\section{References}

1. R.-J. Essiambre, G. Kramer, P. J. Winzer, G. J. Foschini, and B. Goebel, "Capacity limits of optical fiber networks," J. Lightwave Technol., vol. 28, no. 4, pp. 662-701, 2010.

2. C. Koebele, M. Salsi, G. Charlet, and S. Bigo, "Nonlinear effects in mode-division-multiplexed transmission over few-mode optical fiber," IEEE Photon. Technol. Lett., vol. 23, no. 18, pp. 13161318, Sep. 2011.

3. A. Carena, V. Curri, G. Bosco, P. Poggiolini, and F. Forghieri, "Modeling of the impact of nonlinear propagation effects in uncompensated optical coherent transmission links," J. Lightwave Technol., vol. 30, no. 10, pp. 1524-1539, 2012.

4. F. Ferreira, S. Jansen, P. Monteiro, and H. Silva, "Nonlinear semi-analytical model for simulation of few-mode fiber transmission," IEEE Photon. Technol. Lett., vol. 24, no. 4, pp. 240-242, 2012.

5. G. Rademacher, S. Warm, and K. Petermann, "Analytical description of cross-modal nonlinear interaction in mode multiplexed multimode fibers," IEEE Photon. Technol. Lett., vol. 24, no. 21, pp. 1929-1932, 2012.

6. A. E. El-Fiqi, A. Ismail, Z. A. El-Sahn, H. M. H. Shalaby, and R. K. Pokharel, "Evaluation of nonlinear interference in few-mode fiber using the Gaussian noise model," in IEEE Conf. on Lasers and Electro-Optics, (CLEO 2015), to appear, San Jose, CA, May 10-15, 2015.

7. A. Mecozzi, C. Antonelli, and M. Shtaif, "Nonlinear propagation in multi-mode fibers in the strong coupling regime," Opt. express, vol. 20, no. 11, pp. 11673-11678, 2012.

8. G. Bosco, P. Poggiolini, A. Carena, V. Curri, and F. Forghieri, "Analytical results on channel capacity in uncompensated optical links with coherent detection," Opt. Express, vol. 19, no. 26, pp. B440-B451, 2011.

9. I. Kaminow, T. Li, and A. E. Willner, Eds., Optical Fiber Telecommunications Volume VIB, Sixth Edition: Systems and Networks, 6th ed. Amsterdam ; Boston: Academic Press, May 2013. 